\title{
A design tool for the performances comparison of innovative energy systems for naval applications
}

\author{
D.Rattazzi ${ }^{1}$, M. Rivarolo ${ }^{I}$, T. Lamberti ${ }^{1}$, L. Magistri $^{l}$ \\ ${ }^{1}$ Thermochemical Power Group, University of Genoa, via Montallegro 1, 16145 Genoa, Italy
}

\begin{abstract}
This paper aims to develop a tool for the performances comparison of innovative energy systems on board ships, both for concentrated and distributed generation applications. In the first part of the study, the tool database has been developed throughout a wide analysis of the available market solutions in terms of energy generation devices (i.e. fuel cells, internal combustion engines, micro gas turbines), fuels (hydrogen, natural gas, diesel) and related storage technologies. Many of these data have been collected also thanks to the laboratory experience of the authors' research group on different innovative energy systems. From the database, a wide range of maps has been created, correlating costs, volumes, weights and emissions with the installed power and the operational hours required, given by the user as input. The tool highlights the best solution according to the different relevance chosen by the user for each key parameter (i.e. costs, volumes, emissions). In the second part, two different case studies are presented in order to underline how the installed power, the different ship typology and the user requirements affect the choice of the best solution. It is worth noting that the methodology has a general value, as the tool can be applied to both the design of new ships, and to the retrofit of already existing ships in order to respect new requirements (e.g. more and more stringent normative in terms of pollutant emissions in ports and restricted areas). Furthermore, the database can be easily extended to other generation and storage technologies.
\end{abstract}

\author{
Nomenclature \\ $\mathrm{FO}=$ Fuel Oil \\ $\mathrm{IMO}=$ International Maritime Organization \\ $\mathrm{MDO}=$ Marine Diesel Oil \\ $\mathrm{MH}=$ Metal Hydrides
}

\author{
ICE $=$ Internal Combustion Engine \\ $\mathrm{LNG}=$ Liquid Natural Gas \\ $\mathrm{mGT}=$ micro Gas Turbine \\ PEMFC $=$ Proton Exchange Membrane Fuel Cell
}

\section{Introduction}

Nowadays the energy production on board ships and their emissions are one of the most crucial topics in the maritime research field. In fact, the global climate situation is leading the normative companies to address the maritime application to low environmental impact, innovative and sustainable energy systems. Recently, the European Commission put forward a strategy for low-emission mobility, followed in November 2018 by the document "Strategic long-term vision for a prosperous, modern, competitive and climate neutral economy by 2050 - A Clean Planet for all" [1]. The International Maritime Organization (IMO) adopted the Initial Strategy on the reduction of Green House Gas emissions by 2050, aiming at reducing emissions by $50 \%$ by 2050 , compared to 2008 [2]. The introduction of innovative highly efficient technologies will support the transition towards sustainable 
waterborne transport options, capable of significantly emissions reduction [3][4]. Shipping and inland navigation are shifting their power generation towards sustainable options, including renewable energies [5][6]. High efficiency solutions are a worthy solution in order to minimize fuel consumptions, as volumes and weights are significative parameters in maritime systems design; therefore, an accurate evaluation of these characteristics has to be considered to decide the feasibility of innovative technologies on board. Furthermore, costs are a relevant indicator in the design process. In a previous study, the authors investigated different technologies for large scale (more than $5 \mathrm{MW}$ ) power generation on board for large scale vessels [7], finding that Proton Exchange Membrane Fuel Cells (PEMFCs, directly fuelled by pure hydrogen) and micro Gas Turbines (mGTs, fuelled by Natural Gas) can be the most currently suitable innovative technologies. These technologies are compared with the "state of the art technology", represented by Internal Combustion Engines (ICEs), which can be fuelled by different fuel oils or by Natural Gas. Storage systems are taken also into account in order to consider the global performances. For what concerns the hydrogen, the three main storage systems are analysed in this work: Liquid Hydrogen (LH2) tanks, Compressed Hydrogen ( $\mathrm{CH} 2)$ cylinders and Metal Hydrides (MH). Natural Gas is instead considered only as Liquid Natural Gas (LNG) in proper tanks, since it is an already proven system that allows minimizing volumes.

\section{Methodology}

To understand the feasibility of the different technologies for maritime applications, it is important to study the system performance in terms of volumes, weights, costs and emissions. Therefore, for each solutions a deep and detailed market investigation has been performed, considering both storages and power units. Using this strong database, specific maps have been created correlating the main parameters with the size system required. The maps allow for having the overall characteristics for each system: the results will be evaluated in order to provide the most suitable solution for the chosen application considering the parameters relevance set by the users. The flow chart in Figure 1 shows the calculations performed in the tool.

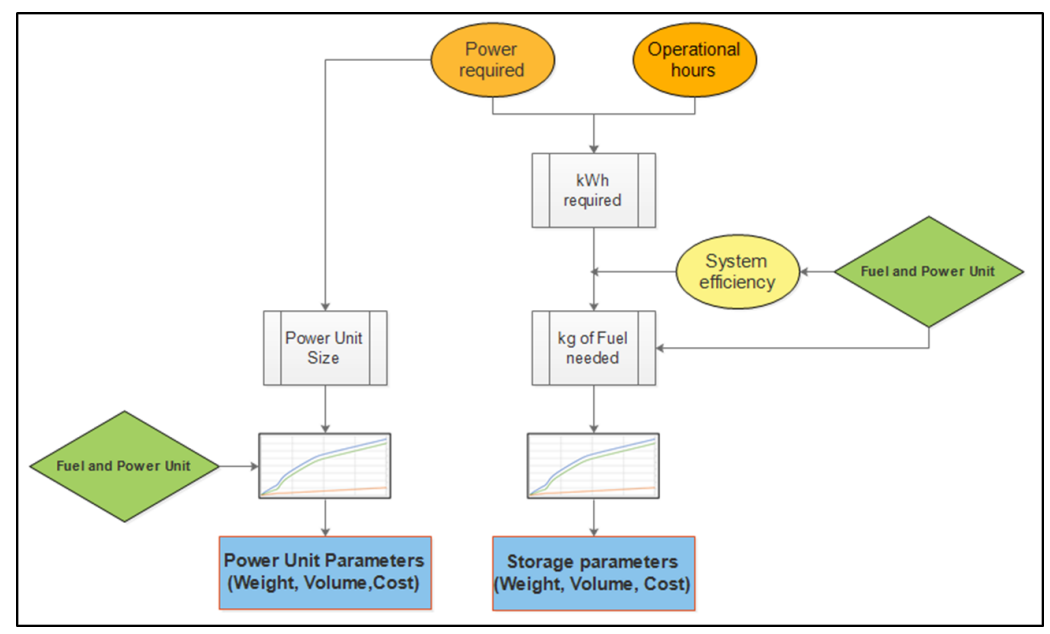

Figure 1 Calculation flow chart

The main inputs are the power required by the ship and the operational hours estimated without docking: their product is the energy amount required by the ship. Dividing this 
energy for the system efficiency, which depends on the technology, the amount of fuel needed is calculated. This value is, in the end, used as an input in the maps to calculate storage parameters (weight, volume and cost). For what concerns the power unit, its size depends on the power required, therefore this value is directly inserted into the power unit map (related to the chosen technology) to obtain the power unit parameters. Emissions are evaluated using maps for each power unit where the input is the quantity of fuel consumed or the power produced.

\subsection{Fuel Storages}

Since the Fuel storage can represent the most relevant component in terms of volumes and weights, it is important to analyse its characteristics. For what concerns the PEMFCs, they are directly fuelled by Hydrogen. Hydrogen is well known for its high LHV (Lower Heating Value) in terms of mass, despite its low energy density in terms of volume. Hence it is important to store it in a high-density form. The options for its storage already available are: (i) Liquid Hydrogen that guarantees a fuel density around $72 \mathrm{~kg} / \mathrm{m} 3$ using cryogenic tanks (at $-252{ }^{\circ} \mathrm{C}$ ); (ii) Compressed $\mathrm{H} 2$ stored in tanks with different density depending on the chosen pressure (i.e. $12 \mathrm{~kg} / \mathrm{m} 3$ at $200 \mathrm{bar}, 35 \mathrm{~kg} / \mathrm{m} 3$ at 700 bar); (iii) Metal Hydrides, which allow for storing large amounts of Hydrogen at ambient pressure, but on the other hand imply a disadvantage in terms of weights. The fuel considered for mGTs supplying is Natural Gas, which has an already developed and well-proven storage technology onboard: Liquefied Natural Gas (LNG). It consists into the use of an insulated tank that guarantees an internal temperature of $-162^{\circ} \mathrm{C}$, allowing for high fuel density (around $450 \mathrm{~kg} / \mathrm{m} 3$ ). Since volume reduction is a very important feature in maritime applications, the storage system considered for natural gas is LNG. As far as Internal Combustion Engines (ICEs) are concerned, two fuels have been evaluated: LNG or Marine Diesel Oil (MDO). The first one represents the most advanced technology to minimize emissions on board ships nowadays; the second one instead is the actual state of the art solution. Marine Diesel Oil is a well-known technology; therefore, a huge database is available, especially for large sizes.

\subsection{Power Units}

The considered power units are PEMFCs, mGTs and ICEs. PEMFCs represent the most promising solutions in an eco-friendly scenario since their zero $\mathrm{CO} 2$ and pollutant emissions; moreover, they are characterized by high level of efficiency, also in off-design conditions. On the other hand, they still present high investment costs compared to traditional engine generators. ICEs, on the opposite, are a very well proven technology that unfortunately leads to significant pollutant emissions that could not be feasible with the more and more stringent normative on this topic. The mGT technology represents a compromise between the two cited above. Indeed, they are a quite well proven technology, even if mainly on heavy-duty applications, and they guarantee lower emissions compared to diesel ICEs (not zero). Considering these aspects, it is evident how a deep consideration in terms of costs, volume and weights for different sizes is necessary to understand which can be the most feasible choice depending on the application.

\section{TPG Tool description}

In order to have a clear vision and a detailed evaluation of the different technologies, a user-friendly design tool has been developed. In this way, it is possible to process the huge database collected during the market investigation using maps and functions that fit the data; elaborating the database is important to understand which is the most suitable application, depending on the different relevance set. Figure 2 shows the software layout. 


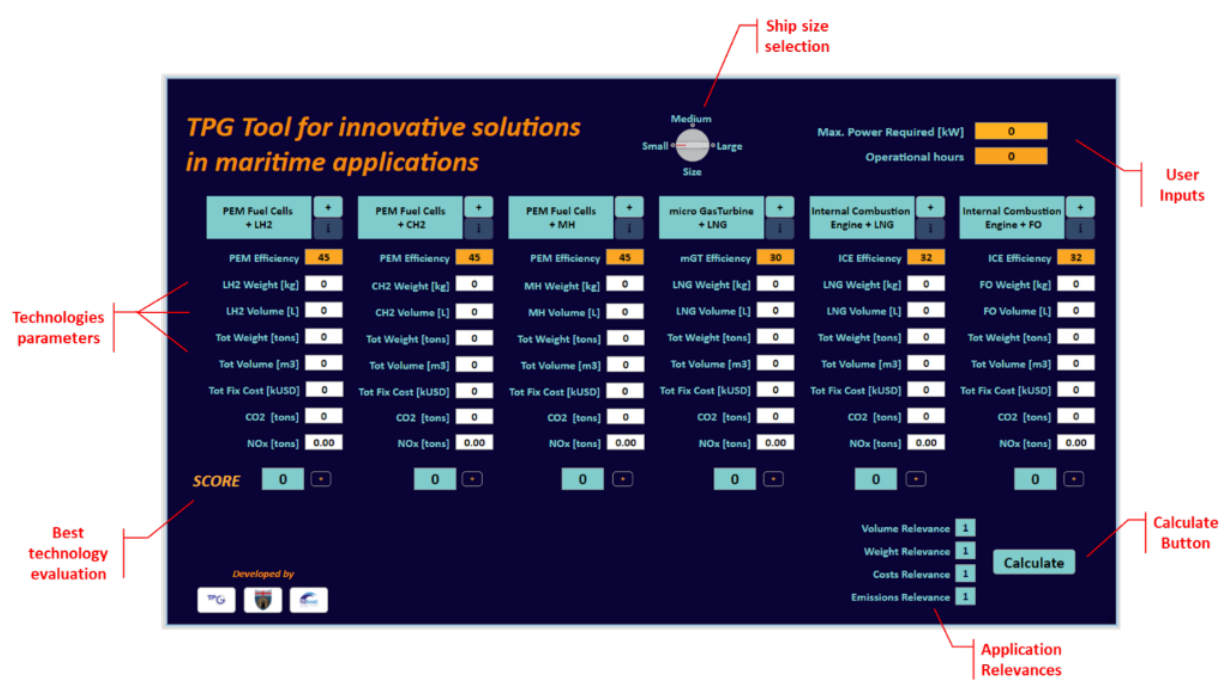

Figure 2 Tool layout description

The cells in orange are editable by the user; hence, the main user inputs are the max power installed on board and the operational hours. The power required is the main input for the power unit design, while the operational hours (hours of navigation without docking) influence the storage design. Another parameter that can be set is the system efficiency: this choice allows the user to set the simulation closer to the system available in its context. The tool provides a default value, representing the average value collected in the market analysis. The knob gives the option to set the boat size in order to understand if a solution is feasible with the considered boat. Pushing the button "Calculate", the data are elaborated and displayed on the screen. The displayed data are the amount of fuel stored (mass and volume), the overall system properties (mass, volume and costs) and the main pollutant emissions $\left(\mathrm{CO}_{2}\right.$ and $\left.\mathrm{NOx}\right)$ that the systems exhaust during the operational hours.

The score represents an evaluation of each technology: higher the score and better the application. The total score for each solution is the sum of single scores obtained for the four categories: volume, weight, cost and emissions. For each category, the score is variable between a minimum of 1 up to a maximum of 10: the technology with the lowest value (i.e. lowest weight) obtains the maximum score; the other technologies are compared with the best one using a parameter $\mathrm{X}$, defined as follows for the considered value (V):

$$
\mathrm{X}=\mathrm{V}_{\mathrm{i}} / \mathrm{V}_{\text {best }}
$$

Where $V_{i}$ represents the value for a generic technology and $V_{\text {best }}$ represents the value for the best solution. The technologies are classified in different ranges, obtaining a corresponding score, as Table 1 shows.

Table 1: Example of scoring (parameter: weight)

\begin{tabular}{|cccc|}
\hline Value & Score & Value & Score \\
\hline $1<\mathrm{X} \leq 1.1$ & 10 & $3<\mathrm{X} \leq 4$ & 5 \\
$1.1<\mathrm{X} \leq 1.3$ & 9 & $4<\mathrm{X} \leq 5$ & 4 \\
$1.3<\mathrm{X} \leq 1.6$ & 8 & $5<\mathrm{X} \leq 6$ & 3 \\
$1.6<\mathrm{X} \leq 2$ & 7 & $6<\mathrm{X} \leq 8$ & 2 \\
$2<\mathrm{X} \leq 3$ & 6 & $\mathrm{X}>8$ & 1 \\
\hline
\end{tabular}


Each application could have specific requirements (i.e. a ship mainly used for navigation in ECAs will consider emissions as the most relevant parameter). The resulting score is affected by the relevance parameters initially set, depending on the considered application. The score for each technology is multiplied for the corresponding relevance (i.e. if weight relevance is 3 , the maximum score for the weight category is 30 points).

Once the calculation is completed, the tool provides also automatic limitations concerning the ship size initially selected. Hence, taking into account the ship dimensions, the tool highlights in red the parameters that are not feasible for the chosen application. It is also possible, clicking on the cyan "plus" button, to have a detailed report about the technology properties with all the data related to storage and power unit. If a detailed analysis of the score is needed, clicking on the blue button "plus", the scores are displayed separately for each relevance parameter. The great versatility standing behind this tool allows easily updating and upgrading it just adding new technologies uploading their maps, representing the most significant parameters.

Errore. L'origine riferimento non è stata trovata. shows an example of the used maps for the Fuel Oil storage. It should be noted that the curves have different trends depending on the storage capacity; this is due to the use of different specific functions extrapolated from real market data. In this case, three different categories of storage have been defined: the first for capacities up to 5000L, the second for capacities between 5000L and $20000 \mathrm{~L}$ and the last for capacities higher than 20000L. Similar different categories have been reported for the other storages and for power units.

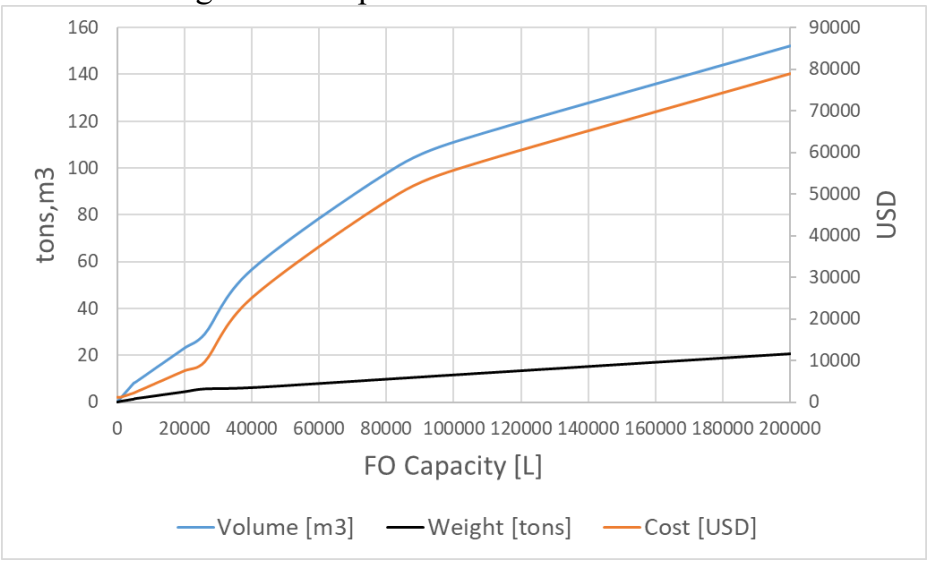

Figure 3 Maps for Fuel Oil storages

\section{Case Studies}

In order to show and test the usefulness of the developed tool, two different real case studies are presented. The case studies are selected to underline how much the relevance parameters required by a specific application affect the best solution. Two different sizes are presented and investigated.

\subsection{Small/Medium size case}

The boat chosen to represent this case study is the Azimut Magellano 76 [8]. This yacht is $24 \mathrm{~m}$ length and 6.13 width with a total weight (full load) of 66 tons. The boat is equipped with two MAN V8 engines for a total power of $1470 \mathrm{~kW}(2000 \mathrm{mHP})$ and has a fuel tank capacity of $7500 \mathrm{~L}$, therefore around 15 hours of navigation without docking can be estimated. Considering the possible main uses and navigation routes for this kind of boat, 
an accurate evaluation of the relevance parameters is performed. In this scenario, the main relevance can be related to the chance to navigate wherever without any issues, especially in touristic zones (most of them are included in ECAs). Volumes represent another significant parameter in terms of comfort: small system volumes allow for larger space for services and rooms. Weights have a lower relevance, while costs are the lowest for yachts. Figure 4 shows the results obtained for this application. The red coloured cells for PEMFC with compressed hydrogen and PEMFC metal hydrides suggest that these technologies are not feasible for the considered scenario, the first one in terms of volume and the second for the weights. Hence these solutions are omitted in the analysis.

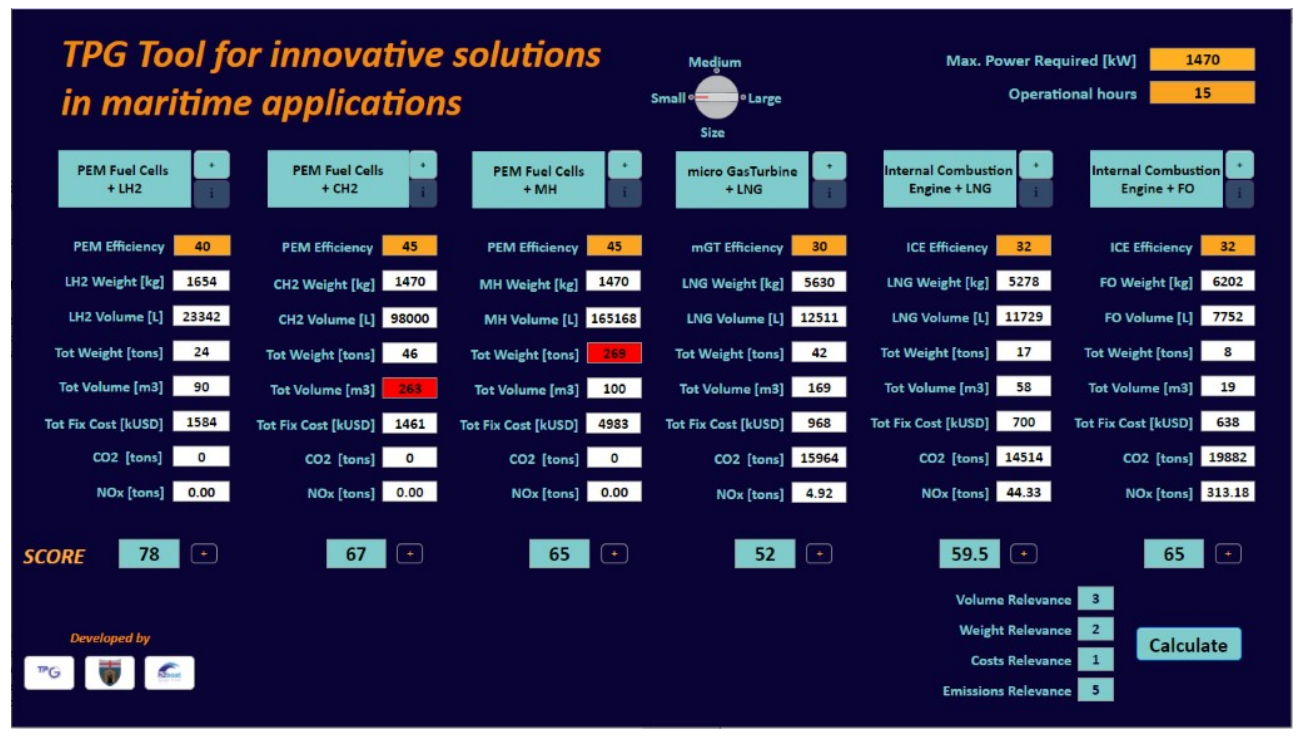

Figure 4 Small/medium size case results

It is worth noting that the systems efficiency for the $\mathrm{PEMFC}+\mathrm{LH} 2$ system has been set to $40 \%$ because, in this way, the energy needed for keeping the tank at cryogenic temperature $\left(-253^{\circ} \mathrm{C}\right)$ is taken into proper account. Looking at the scores it is possible to note that the most performant solution is the one with PEMFCs+LH2. The second acceptable solution is $\mathrm{ICE}+\mathrm{FO}$, which represents the state of the art; the use of LNG with mGTs or ICEs leads to worse results. The main advantage given by the use of PEMFC is certainly its zero emissions, leading to great advantages in terms of free navigation areas. Moreover, storing $\mathrm{H} 2$ in a liquid form it is possible to minimize the system volumes and weights being quite competitive with the solutions with ICEs. The use of mGT in this application is the less competitive because of large weights and

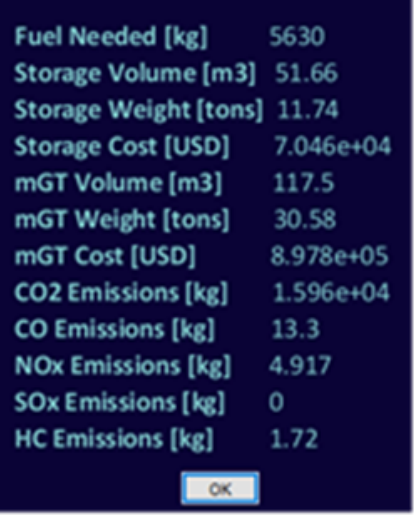

Figure 5 mGT parameters detail volumes (see Figure 5). Indeed in this scenario the power units (high value for the required power and few operational hours) mainly affect the results. Considering this aspect, the mGTs are the power units with highest volume and weight values. It is also possible to see how the use of LNG (especially with mGT) allows for very low values of NOx emissions. Comparing the ICEs solutions, the one with LNG has lower values for the emissions but, 
on the other hand, the LNG storage system has higher volumes and weights if compared with MDO. As expected, PEMFCs have the higher costs: looking at the other cost values, the most developed technologies for maritime application (ICEs) have lower costs.

\subsection{Large size case}

The ship considered for the large size case is the Viking Sky ferry built by Fincantieri [9]. This ferry has a length of $228 \mathrm{~m}$ and a moulded beam of $28.8 \mathrm{~m}$ with a total gross weight of 47800 tons. The ferry is equipped with two MAN 9L32/44CR engines and two MAN $12 \mathrm{~V} 32 / 44 \mathrm{CR}$ engines for a total installed electric power of $23.5 \mathrm{MW}$. The propulsion requests around $14.5 \mathrm{MW}$, hence it can be approximately considered that the electrical loads related to hotel services and supply systems have a peak value of $9 \mathrm{MW}$. The operational hours for this application can be assumed about 24 hours. In this application the innovative technologies are considered only to cover the loads referred to hotel services and supply systems. This choice derives from the need of reduce pollutant emissions mainly in ports, during the docking period, when the propulsion load is zero. The ferry applications have different needs and requirements respect to the small/medium size case. In this context emissions are still a significant parameter but not preponderant on volumes. Indeed in ferry applications is extremely important to have most space as possible for rooms and for commercial onboard activities like restaurants and bars. Therefore, these two aspects are considered as the most relevant for this scenario. From an economic point of view, costs cannot be neglected, while weights do not significantly affect the choice of the best solution. The overall efficiencies for FC systems have been increased if compared with the small/medium case because the systems for this application are usually significantly oversized; therefore, fuel cells work mainly at partial loads, where their efficiency is higher [7]. The simulation provided by the tool suggests LNG ICE as best solution (total score 69): this is, in fact, the best compromise in terms of emissions, volumes and costs. All the studied technologies are feasible with the considered application even if the use of compressed $\mathrm{H} 2$ and metal hydrides is not very competitive with the other technologies. Figure 6 shows the score details.

\begin{tabular}{|c|c|c|c|c|c|c|}
\hline A. PEM+LH2 Score Detail & $\times$ & A ICE+LNG Score Detail & $\square$ & A ICE+FO Score Detail & $\square$ & $x$ \\
\hline VOLUME SCORE: & 12 & VOLUME SCORE: & 24 & VOLUME SCORE: & 30 & \\
\hline WEIGHT SCORE: & 5 & WEIGHT SCORE: & 7 & WEIGHT SCORE: & 10 & \\
\hline COSTS SCORE: & 10 & COSTS SCORE: & 20 & COSTS SCORE: & 20 & \\
\hline CO2 EMISSIONS SCORE: & 20 & CO2 EMISSIONS SCORE: & 12 & CO2 EMISSIONS SCORE: & 2 & \\
\hline NOX EMISSIONS SCORE: & 20 & NOX EMISSIONS SCORE: & 6 & NOX EMISSIONS SCORE: & 2 & \\
\hline OK & & OK & & OK & & \\
\hline
\end{tabular}

Figure 6 Score details for the main solutions

PEMFC+LH2 application (total score 67) is the best in terms of emissions without being significantly competitive in terms costs and volumes, while the use of ICE+FO (total score 64 ) is the most performant in terms of volumes, weights and costs but it is significantly penalized in terms of emissions. Using $\mathrm{ICE}+\mathrm{LNG}$ it is possible to obtain a good compromise.

\section{Conclusions}

In this paper a tool for the design of innovative power units on board ships has been presented. After a first general description of the tool, two case studies have been analysed 
in order to show the tool behaviour. The results obtained during with these examples are feasible with precedent studies also provided by the authors. Therefore a reliable behaviour of the tool can be assumed for the presented technologies. In add, it can be considered a tool upgrade with other innovative systems like Solid Oxide Fuel Cells (SOFCs), even if by now the available values and data have mainly a research field source and not a real market one. It is worth to notice that the tool can be used in a preliminary level in order to understand which technology can be considered firstly. After that, it will be necessary to analyse more in detail the ship structure to understand where the energy storages and the power units can be placed on board. Moreover, especially for the hydrogen case, some specific risks analysis has to be performed for health and safety reasons. In the end, this tool can be considered as the beginning step of ship designs, with the benefit of userfriendly graphics that make it easy to be used also by basic users. In the end, we can assume that the work standing behind the tool guarantees an enormous time saving for all the next activities on this topic, representing a strong base for the future studies about innovative energy systems on maritime applications.

\section{References}

[1] http://europa.eu/rapid/press-release_IP-18-6543_en.htm, access 30/01/2019.

[2] https://www.theicct.org/publications/IMO-initial-GHG-strategy, access 30/01/2019.

[3] A. Armellini, S Daniotti, P Pinamonti, M Reini, "Evaluation of gas turbines as alternative energy production systems for a large cruise ship to meet new maritime regulations", Applied Energy, 211 (2018), 306-317.

[4] R Tang, X Li, J Lai, “A novel optimal energy-management strategy for a maritime hybrid energy system based on large-scale global optimization", Applied Energy, 228 (2018), 254-264.

[5] L van Biert, M Godjevac, K Visser, P V Aravind, “A review of fuel cell systems for maritime applications", Journal of Power Sources, 327(2016), 345-364.

[6] R D Geertsma, R R Negenborn, K Visser, J J Hopman, "Design and control of hybrid power and propulsion systems for smart ships: A review of developments", Applied Energy, 194 (2017), 30-54.

[7] M Rivarolo, D Rattazzi, L Magistri, "Best operative strategy for energy management of a cruise ship employing different distributed generation technologies", International Journal of Hydrogen Energy, 43 (2018), 23500-235210.

[8] https://azimutyachts.com/azimutmagellano76.html, access 27/02/2019

[9] https://www.fincantieri.com/it/prodotti-servizi/navi-crociera/viking-sky/, access $27 / 02 / 2019$ 